\title{
Mental Health Status Among Frontline Prison Police During The Prevention and Control of The COVID-19 Epidemic: A Cross-Sectional Survey From China
}

\author{
Yong Tian \\ Renmin University of China \\ Tingyong Feng \\ Southwest University \\ Zhen Wen \\ Chengdu Third People's Hospital \\ Yipeng Yang \\ Sichuan Judicial and Police Officers Peofessional College \\ Jingting Huang ( $\nabla$ hjt897832534@163.com ) \\ Sichuan University
}

\section{Research Article}

Keywords: COVID-19, Mental health, Frontline prison police, Prevention and control mode

Posted Date: June 17th, 2021

DOl: https://doi.org/10.21203/rs.3.rs-569644/v1

License: (c) (i) This work is licensed under a Creative Commons Attribution 4.0 International License. Read Full License 


\section{Abstract \\ Background}

A global public health emergency triggered by the Coronavirus Disease 2019 (COVID-19) epidemic may have a remarkable psychological impact on the population. There is still limited psychological research on police officers, especially on prison police. The present study aims to identify prevalence and influencing factors on mental health status among frontline prison police in China during the prevention and control of the COVID-19 epidemic.

\section{Methods}

A cross-sectional survey with a sample of 981 frontline prison police was conducted by using snowball sampling approach. The self-administered questionnaire consisted of 4 parts: (i) informed consent form; (ii) socio-demographic section; (iii) work and life situations during the prevention and control of the COVID-19 epidemic; (iv) the Chinese version of the 12-item General Health Questionnaire (GHQ-12). Univariate analysis and multivariable logistic regression were performed to identify factors influencing mental health status.

\section{Results}

The prevalence of being prone to mental health problems (GHQ-12 score $\geq 4$ ) was $33.43 \%$ among frontline prison police. The results of GHQ-12 factors analysis that the prison police suffered from psychological issues related to anxiety and depression, which main symptoms were unhappy and depressed, lost sleep over worry and constantly under strain. Multivariate logistic regression analysis revealed that male $(\mathrm{OR}=1.573,95 \% \mathrm{Cl}: 1.385-1.853)$, work in closed jail $(\mathrm{OR}=2.203,95 \% \mathrm{Cl}: 2.139-$ 2.297), more night shifts ( $\mathrm{OR}=2.163,95 \% \mathrm{Cl}: 2.031-2.317 ; \mathrm{OR}=2.749,95 \% \mathrm{Cl}: 2.194-2.901)$, more smoking $(\mathrm{OR}=1.100,95 \% \mathrm{Cl}: 1.037-2.168)$, poor physical condition $(\mathrm{OR}=1.947,95 \% \mathrm{Cl}: 1.478-2.250)$, chronic or serious illness history $(\mathrm{OR}=1.870,95 \% \mathrm{Cl}: 1.314-2.660 ; \mathrm{OR}=2.214,95 \% \mathrm{Cl}: 1.460-2.812)$ were risk factor for mental health among frontline prison police, while regular diet $(\mathrm{OR}=0.779,95 \% \mathrm{Cl}$ : $0.539-0.928)$, more physical exercise $(\mathrm{OR}=0.702,95 \% \mathrm{Cl}: 0.548-0.899 ; \mathrm{OR}=0.641,95 \% \mathrm{Cl}: 0.316-$ $0.887)$, more communication with family members $(\mathrm{OR}=0.437,95 \% \mathrm{Cl}: 0.295-0.616)$ were protective factor.

\section{Conclusion}

The frontline prison police exhibited a high tendency toward mental health problems during the prevention and control of this epidemic. Therefore, continued surveillance of psychological problems and targeted mental health care for frontline prison police were urgently. 


\section{Background}

Since the late December 2019, the 2019 Coronavirus Disease (COVID-19) has spread to all countries, areas or territories of the world. On January 30,2020, the epidemic was declared a global public health emergency by the WHO Emergency Committee [1]. There have been more than 100 million confirmed cases, including 2,147,411 deaths were reported worldwide as of January 26, 2021 [2]. Countries took different safety measures and precautions to limit the spread and risk of the epidemic, such as obligatory lockdowns, travel was halted, airports were shut down, many work spaces, schools and universities were closed, and prison visits were cancelled $[3,4]$. The crisis of public health triggered by epidemic and the preventive measures taken to control it have become the major threats to both physical and mental health of the population, especially to frontline staffs $[5,6]$.

As a result of the rapidly spread of the epidemic, prisons in several countries have experienced a serious problem with COVID-19 infection. For example, confirmed cases were reported in prison of British, France, United States, Pakistani, India and Brazil, etc [7-11]. Lessons from COVID-19 infections in both domestic and foreign prisons were learned deeply by the penitentiary system in China. The prison authorities have formulated and implemented many effective measures to prevent and control the epidemic, and achieved major results of no infections, no outbreaks, and no deaths. This achievement was obtained mainly from the perseverance and dedication of frontline prison police. The work patterns of prison police have been divided into three types during the prevention and control of the COVID-19 epidemic, which were work in closed jail for 14 days, centralized isolation and preparation for 14 days, and home quarantine and preparation for 14 days [12]. The stressful task of preventing the epidemic and supervising criminals placed a huge burden on frontline prison police.

During pandemic outbreaks, the frontline warriors including healthcare workers and police staffs were under unprecedented pressure from the heavy workload, risky workplace environment, and nonavailability of adequate leaves/duty off periods $[5,13]$. These severe situations caused them to be especially vulnerable to mental health issues, such as anxiety and depression symptoms $[14,15]$. The psychological problems would negatively interfere with the efficiency of frontline warriors to fight the epidemic, and may pose a threat to their overall well-being. Previous studies have acknowledged concerns about the psychological problems of frontline doctors, nurses and paramedical staffs $[5-6,15]$, and the psychological assistance for healthcare workers have been provided by many countries [16]. A latest study also showed that COVID-19 duties during the lockdown period of police personnel exhibited significant anxiety, depression symptoms, and perceived significant stress [13]. However, there is still no systematic research involving the mental health of prison police.

A cross-sectional survey has been conducted to investigated the psychological issues among frontline prison police during the prevention and control of the COVID-19 epidemic. The aim of the current study was also to explore which factors may influence the mental health of prison police. These findings will aid local governments and prison administrators to formulate effective policies, regulations and 
actionable psychological interventions for promoting mental wellbeing of frontline prison police in public health emergencies.

\section{Methods}

\section{Study design and participants}

This cross-sectional study was implemented using a anonymous web-based survey from March 28 to April 20, 2020. The purpose of the survey lasting for nearly a month was to investigate as much as possible subjects who have experienced all work patterns during the prevention and control of the COVID19 epidemic. The participants of present study comprised part of frontline prison police in western China. Those who have been diagnosed with a history of mental disorder, refused to participate in survey, and unable to operate smart phone or computer devices were excluded.

\section{Procedure and survey questionnaire}

In order to minimize face-to-face communication and contact, recruitment of participants was done using snowball sampling approach. The survey was executed through an online survey platform called "Questionnaire Star" (Changsha Ranxing Science and Technology, Shanghai, China). The questionnaire link was circulated by WeChat or other social tools, after seeking permission from the prison authorities. Finally, a total of 1035 questionnaires were completed without assistance, of which 981 questionnaires were valid.

The survey questionnaire was developed with 4 parts of informed consent, socio- demographic factors, items on work and life situation of frontline prison police, and mental health status. (i) Participants were informed that the right to take a voluntary participation was absolute, and the completion of the questionnaire imply providing the consent. (ii) Demographic characteristics of the participants included variables such as gender, age, marital status, education, and working years, etc. (iii) A self-designed questionnaire was used to collected the informations about recent work mode, number of night shifts, diet, physical exercise, smoking, physical condition, disease history, and communication with family members. (iv) The Chinese version of the 12-item General Health Questionnaire (GHQ-12) was used to measure mental health status in present study [17]. The self-administered scale composed of 12 items with answers scored from "better than usual / not at all = 0" and "same as usual / no more than usual = 0 " to "less than usual / rather more than usual $=1$ " and "much less than usual / much more than usual = 1" [18]. All item scores were are added to give GHQ-12 total score, which the possible score range from 0 to 12. The scale with higher total scores indicating higher degrees of disturbance of the mental health status [19]. In this study, the GHQ-12 was reliable and repeatable and showed good internal consistency (Cronbach's alpha was 0.895). An additional table file shows this questionnaire in more detail [see Additional file 1].

\section{Ethical considerations}


This study protocol was approved by the Ethical Review Board of West China Medical School at Sichuan University. All participants have signed the informed consent form via the "Questionnaire Star" after explaining the purpose of the study. Their personal information was collected anonymously, and all data were used for research purposes only.

\section{Statistical analysis}

Statistical analysis was performed with SPSS 24.0 software (SPSS Inc, Chicago, IL, USA). Enumeration data were presented as the numbers $(n)$ and percentages (\%), and compared between individuals with and those without tendency of mental health problems using chi-squared $\left(\chi^{2}\right)$ test for categorical variables. Measurement data of the GHQ-12 score were computed using the means (SD). Multivariable logistic regression was performed to determine the association of multiple potential predictors (independent variables, seted as $\mathrm{X}$ ) with mental health (dependent variable, seted as $\mathrm{Y}$ ) by calculating the odds ratios and their $95 \%$ confidence interval. The independent variables that were significant by $\chi^{2}$ test were selected, and mental health status $(Y=1$, with tendency of psychological problems; $Y=0$, without tendency of psychological problems) was the dependent variable. Data were considered statistically significant at the level of a $p$-value less than 0.05 .

\section{Results}

\section{General characteristics of the participants}

A total of 981 frontline prison police, including $262(26.71 \%)$ of female and $719(73.29 \%)$ of male, participated in this study with the response rate of $94.78 \%$. The age of the respondents range from 20 to 57 years, and the mean age was $(29.56 \pm 6.13)$ years. The marital status of being married $(46.59 \%)$ was similar to that of being single (53.41\%). Majority of the sample had a bachelor degree and above (73.80\%), and $26.20 \%$ had a college degree and below. $55.56 \%$ have been working less than 5 years in their profession, followed by between 5 years and 10 years $(30.17 \%)$. Table 1 presented the general characteristics of the participants, and the groups significantly differed on gender, working years, recent work mode, number of night shifts, diet, physical exercise, smoking, physical condition, disease history in the past year, and communication with family members (all $p$-value $<0.05$ ). 
Table 1

Characteristics and mental health status of frontline prison police $[n(\%)]$.

\begin{tabular}{|c|c|c|c|c|c|c|}
\hline \multirow[t]{2}{*}{ Variables } & \multirow[t]{2}{*}{ Grouping } & \multirow[t]{2}{*}{$\begin{array}{l}\text { Total } \\
\text { sample }\end{array}$} & \multicolumn{2}{|c|}{$\begin{array}{l}\text { Tendency of mental } \\
\text { health problems }\end{array}$} & \multirow[t]{2}{*}{$x^{2}$} & \multirow[t]{2}{*}{$p$-value } \\
\hline & & & $\begin{array}{l}\text { With } \\
\text { (GHQ-12 } \\
\text { score } \geq \\
\text { 4) }\end{array}$ & $\begin{array}{l}\text { Without } \\
(\text { GHQ-12 } \\
\text { score < 4) }\end{array}$ & & \\
\hline \multirow[t]{2}{*}{ Gender } & Female & $\begin{array}{l}262 \\
(26.71 \%)\end{array}$ & $\begin{array}{l}70 \\
(26.72 \%)\end{array}$ & $\begin{array}{l}192 \\
(73.28 \%)\end{array}$ & 6.435 & $\star 0.011$ \\
\hline & Male & $\begin{array}{l}719 \\
(73.29 \%)\end{array}$ & $\begin{array}{l}254 \\
(35.33 \%)\end{array}$ & $\begin{array}{l}465 \\
(64.67 \%)\end{array}$ & & \\
\hline \multirow[t]{4}{*}{ Age (years) } & $<30$ & $\begin{array}{l}692 \\
(70.54 \%)\end{array}$ & $\begin{array}{l}225 \\
(32.51 \%)\end{array}$ & $\begin{array}{l}467 \\
(67.49 \%)\end{array}$ & 2.202 & 0.333 \\
\hline & $30-50$ & $\begin{array}{l}241 \\
(24.57 \%)\end{array}$ & $\begin{array}{l}91 \\
(37.76 \%)\end{array}$ & $\begin{array}{l}150 \\
(62.24 \%)\end{array}$ & & \\
\hline & $>50$ & $\begin{array}{l}48 \\
(4.89 \%)\end{array}$ & $\begin{array}{l}16 \\
(33.33 \%)\end{array}$ & $\begin{array}{l}32 \\
(66.67 \%)\end{array}$ & & \\
\hline & Mean $\pm S D$ & $\begin{array}{l}29.56 \pm \\
6.13\end{array}$ & & & & \\
\hline \multirow[t]{2}{*}{ Marital status } & Married & $\begin{array}{l}457 \\
(46.59 \%)\end{array}$ & $\begin{array}{l}151 \\
(33.04 \%)\end{array}$ & $\begin{array}{l}306 \\
(66.96 \%)\end{array}$ & 0.060 & 0.807 \\
\hline & Single & $\begin{array}{l}524 \\
(53.41 \%)\end{array}$ & $\begin{array}{l}177 \\
(33.78 \%)\end{array}$ & $\begin{array}{l}347 \\
(66.22 \%)\end{array}$ & & \\
\hline \multirow[t]{2}{*}{ Education } & $\begin{array}{l}\text { College degree } \\
\text { and below }\end{array}$ & $\begin{array}{l}257 \\
(26.20 \%)\end{array}$ & $\begin{array}{l}96 \\
(37.35 \%)\end{array}$ & $\begin{array}{l}161 \\
(62.65 \%)\end{array}$ & 2.403 & 0.121 \\
\hline & $\begin{array}{l}\text { Bachelor } \\
\text { degree and } \\
\text { above }\end{array}$ & $\begin{array}{l}724 \\
(73.80 \%)\end{array}$ & $\begin{array}{l}232 \\
(32.04 \%)\end{array}$ & $\begin{array}{l}492 \\
(67.96 \%)\end{array}$ & & \\
\hline \multirow[t]{3}{*}{ Working years } & $<5$ & $\begin{array}{l}545 \\
(55.56 \%)\end{array}$ & $\begin{array}{l}233 \\
(42.75 \%)\end{array}$ & $\begin{array}{l}312 \\
(57.25 \%)\end{array}$ & 8.460 & $\star 0.015$ \\
\hline & $5-10$ & $\begin{array}{l}296 \\
(30.17 \%)\end{array}$ & $\begin{array}{l}104 \\
(35.14 \%)\end{array}$ & $\begin{array}{l}192 \\
(64.86 \%)\end{array}$ & & \\
\hline & $>10$ & $\begin{array}{l}140 \\
(14.27 \%)\end{array}$ & $\begin{array}{l}44 \\
(31.43 \%)\end{array}$ & $\begin{array}{l}96 \\
(68.57 \%)\end{array}$ & & \\
\hline $\begin{array}{l}\text { Recent working } \\
\text { mode }\end{array}$ & $\begin{array}{l}\text { Home } \\
\text { quarantine } \\
\text { and } \\
\text { preparation }\end{array}$ & $\begin{array}{l}189 \\
(19.27 \%)\end{array}$ & $\begin{array}{l}20 \\
(10.58 \%)\end{array}$ & $\begin{array}{l}169 \\
(89.42 \%)\end{array}$ & 167.576 & $\begin{array}{l}\star *< \\
0.001\end{array}$ \\
\hline
\end{tabular}




\begin{tabular}{|c|c|c|c|c|c|c|}
\hline \multirow[t]{2}{*}{ Variables } & \multirow[t]{2}{*}{ Grouping } & \multirow[t]{2}{*}{$\begin{array}{l}\text { Total } \\
\text { sample }\end{array}$} & \multicolumn{2}{|c|}{$\begin{array}{l}\text { Tendency of mental } \\
\text { health problems }\end{array}$} & \multirow[t]{2}{*}{$x^{2}$} & \multirow[t]{2}{*}{$p$-value } \\
\hline & & & $\begin{array}{l}\text { With } \\
\text { (GHQ-12 } \\
\text { score } \geq \\
\text { 4) }\end{array}$ & $\begin{array}{l}\text { Without } \\
\text { (GHQ-12 } \\
\text { score < 4) }\end{array}$ & & \\
\hline & $\begin{array}{l}\text { Centralized } \\
\text { isolation and } \\
\text { preparation }\end{array}$ & $\begin{array}{l}379 \\
(38.63 \%)\end{array}$ & $\begin{array}{l}77 \\
(20.32 \%)\end{array}$ & $\begin{array}{l}302 \\
(79.68 \%)\end{array}$ & & \\
\hline & $\begin{array}{l}\text { Work in closed } \\
\text { jail }\end{array}$ & $\begin{array}{l}413 \\
(42.10 \%)\end{array}$ & $\begin{array}{l}231 \\
(55.93 \%)\end{array}$ & $\begin{array}{l}182 \\
(44.07 \%)\end{array}$ & & \\
\hline \multirow[t]{3}{*}{$\begin{array}{l}\text { Number of night } \\
\text { shifts (times/month) }\end{array}$} & $<7$ & $\begin{array}{l}573 \\
(58.41 \%)\end{array}$ & $\begin{array}{l}138 \\
(24.08 \%)\end{array}$ & $\begin{array}{l}435 \\
(75.92 \%)\end{array}$ & 58.459 & $\begin{array}{l}* *< \\
0.001\end{array}$ \\
\hline & $7-15$ & $\begin{array}{l}296 \\
(30.17 \%)\end{array}$ & $\begin{array}{l}129 \\
(43.58 \%)\end{array}$ & $\begin{array}{l}167 \\
(56.42 \%)\end{array}$ & & \\
\hline & $>15$ & $\begin{array}{l}112 \\
(11.42 \%)\end{array}$ & $\begin{array}{l}61 \\
(54.46 \%)\end{array}$ & $\begin{array}{l}51 \\
(45.54 \%)\end{array}$ & & \\
\hline \multirow[t]{2}{*}{ Diet } & irregular & $\begin{array}{l}249 \\
(25.38 \%)\end{array}$ & $\begin{array}{l}127 \\
(51.00 \%)\end{array}$ & $\begin{array}{l}122 \\
(49.00 \%)\end{array}$ & 46.280 & $\begin{array}{l}\star \star \\
0.001\end{array}$ \\
\hline & regular & $\begin{array}{l}732 \\
(74.62 \%)\end{array}$ & $\begin{array}{l}201 \\
(27.46 \%)\end{array}$ & $\begin{array}{l}531 \\
(72.54 \%)\end{array}$ & & \\
\hline \multirow[t]{3}{*}{$\begin{array}{l}\text { Physical exercise } \\
\text { (days/week) }\end{array}$} & No exercise & $\begin{array}{l}633 \\
(64.53 \%)\end{array}$ & $\begin{array}{l}235 \\
(37.12 \%)\end{array}$ & $\begin{array}{l}398 \\
(62.88 \%)\end{array}$ & 10.972 & $\star \star 0.004$ \\
\hline & $\leq 2$ & $\begin{array}{l}232 \\
(23.65 \%)\end{array}$ & $\begin{array}{l}61 \\
(26.29 \%)\end{array}$ & $\begin{array}{l}171 \\
(73.71 \%)\end{array}$ & & \\
\hline & $\geq 3$ & $\begin{array}{l}116 \\
(11.82 \%)\end{array}$ & $\begin{array}{l}32 \\
(27.59 \%)\end{array}$ & $\begin{array}{l}84 \\
(72.41 \%)\end{array}$ & & \\
\hline \multirow[t]{3}{*}{ Smoking } & Never & $\begin{array}{l}499 \\
(50.87 \%)\end{array}$ & $\begin{array}{l}147 \\
(29.46 \%)\end{array}$ & $\begin{array}{l}352 \\
(70.54 \%)\end{array}$ & 14.548 & $\star \star 0.001$ \\
\hline & Sometimes & $\begin{array}{l}253 \\
(25.79 \%)\end{array}$ & $\begin{array}{l}81 \\
(32.02 \%)\end{array}$ & $\begin{array}{l}172 \\
(67.98 \%)\end{array}$ & & \\
\hline & Everyday & $\begin{array}{l}229 \\
(23.34 \%)\end{array}$ & $\begin{array}{l}100 \\
(43.67 \%)\end{array}$ & $\begin{array}{l}129 \\
(56.33 \%)\end{array}$ & & \\
\hline \multirow[t]{2}{*}{ Physical condition } & good & $\begin{array}{l}846 \\
(86.24 \%)\end{array}$ & $\begin{array}{l}219 \\
(25.89 \%)\end{array}$ & $\begin{array}{l}627 \\
(74.11 \%)\end{array}$ & 157.401 & \\
\hline & poor & $\begin{array}{l}135 \\
(13.76 \%)\end{array}$ & $\begin{array}{l}109 \\
(80.74 \%)\end{array}$ & $\begin{array}{l}26 \\
(19.26 \%)\end{array}$ & & \\
\hline $\begin{array}{l}\text { Disease history in } \\
\text { the past year }\end{array}$ & $\begin{array}{l}\text { No or minor } \\
\text { illness }\end{array}$ & $\begin{array}{l}830 \\
(84.61 \%)\end{array}$ & $\begin{array}{l}241 \\
(29.04 \%)\end{array}$ & $\begin{array}{l}589 \\
(70.96 \%)\end{array}$ & 52.960 & $\begin{array}{l}* *< \\
0.001\end{array}$ \\
\hline
\end{tabular}




\begin{tabular}{|c|c|c|c|c|c|c|}
\hline \multirow[t]{2}{*}{ Variables } & \multirow[t]{2}{*}{ Grouping } & \multirow[t]{2}{*}{$\begin{array}{l}\text { Total } \\
\text { sample }\end{array}$} & \multicolumn{2}{|c|}{$\begin{array}{l}\text { Tendency of mental } \\
\text { health problems }\end{array}$} & \multirow[t]{2}{*}{$x^{2}$} & \multirow[t]{2}{*}{$p$-value } \\
\hline & & & $\begin{array}{l}\text { With } \\
\text { (GHQ-12 } \\
\text { score } \geq \\
\text { 4) }\end{array}$ & $\begin{array}{l}\text { Without } \\
(\text { GHQ-12 } \\
\text { score < 4) }\end{array}$ & & \\
\hline & chronic & $\begin{array}{l}133 \\
(13.56 \%)\end{array}$ & $\begin{array}{l}72 \\
(54.14 \%)\end{array}$ & $\begin{array}{l}61 \\
(45.86 \%)\end{array}$ & & \\
\hline & serious illness & $\begin{array}{l}18 \\
(1.83 \%)\end{array}$ & $\begin{array}{l}15 \\
(83.33 \%)\end{array}$ & $\begin{array}{l}3 \\
(16.67 \%)\end{array}$ & & \\
\hline \multirow{2}{*}{$\begin{array}{l}\text { Communication with } \\
\text { family members } \\
\text { (times/week) }\end{array}$} & $0-2$ & $\begin{array}{l}273 \\
(27.83 \%)\end{array}$ & $\begin{array}{l}125 \\
(45.79 \%)\end{array}$ & $\begin{array}{l}148 \\
(54.21 \%)\end{array}$ & 25.932 & $\begin{array}{l}\star \star \\
0.001\end{array}$ \\
\hline & $\geq 3$ & $\begin{array}{l}708 \\
(72.17 \%)\end{array}$ & $\begin{array}{l}203 \\
(28.67 \%)\end{array}$ & $\begin{array}{l}505 \\
(71.33 \%)\end{array}$ & & \\
\hline
\end{tabular}

\section{Prevalence of self-reported mental health problem}

Previous studies have demonstrated that GHQ-12 total scores of four or above indicated tendency of mental health problems in mainland China $[20,21]$. To investigate the prevalence of psychological problems, the self-reported survey was conducted using GHQ-12 scale. The result found that one-third of frontline prison police may have a tendency toward mental health problems during the prevention and control of the COVID-19 epidemic (33.43\%, 328/981). The distribution of the GHQ-12 score was shown in Fig. 1, which was not normally distributed by Kolmogorov-Smirnov test ( $p$-value $<0.05)$.

\section{Psychological factors among frontline prison police}

Many factor-analytic studies have suggested that the GHQ-12 could be divided into three factors, including anxiety and depression (Factor I, item of 2, 5, 6, and 9), social dysfunction (Factor II, item of 1, 3, $4,7,8$, and 12), and loss of confidence (Factor III, item of 10 and 11) [22, 23]. As shown in Table 2, the mean scores were ranked higher with Factor I than with Factor II and III. Meanwhile, the highest total scores were for item 9, 2, and 5. These results indicated that the frontline prison police suffered from psychological problems associated with anxiety and depression, which main symptoms were unhappy and depressed, lost sleep over worry and constantly under strain. 
Table 2

Psychological factors of the 12-item General Health Questionnaire.

\begin{tabular}{|c|c|c|c|c|}
\hline $\begin{array}{l}\text { Psychological } \\
\text { factors }\end{array}$ & Items & $\begin{array}{l}\text { Total } \\
\text { scores }\end{array}$ & $\begin{array}{l}\text { Mean score of } \\
\text { each item }\end{array}$ & $\begin{array}{l}\text { Mean score of } \\
\text { each factor }\end{array}$ \\
\hline \multirow{4}{*}{$\begin{array}{l}\text { Factor I: anxiety and } \\
\text { depression }\end{array}$} & 2. Lost sleep over worry & 453 & 0.46 & 352.5 \\
\hline & $\begin{array}{l}\text { 5. Felt constantly under } \\
\text { strain }\end{array}$ & 414 & 0.42 & \\
\hline & $\begin{array}{l}\text { 6. Felt couldn't overcome } \\
\text { difficulties }\end{array}$ & 56 & 0.06 & \\
\hline & $\begin{array}{l}\text { 9. Been feeling unhappy } \\
\text { and depressed }\end{array}$ & 487 & 0.50 & \\
\hline \multirow{6}{*}{$\begin{array}{l}\text { Factor Il: social } \\
\text { dysfunction }\end{array}$} & 1. Able to concentrate & 188 & 0.19 & 173.5 \\
\hline & $\begin{array}{l}\text { 3. Felt playing useful part } \\
\text { in things }\end{array}$ & 115 & 0.12 & \\
\hline & $\begin{array}{l}\text { 4. Felt capable of making } \\
\text { decisions }\end{array}$ & 103 & 0.10 & \\
\hline & $\begin{array}{l}\text { 7. Able to enjoy day-to- } \\
\text { day activities }\end{array}$ & 293 & 0.30 & \\
\hline & $\begin{array}{l}\text { 8. Been able to face } \\
\text { problems }\end{array}$ & 35 & 0.04 & \\
\hline & $\begin{array}{l}\text { 12. Been feeling } \\
\text { reasonably happy }\end{array}$ & 307 & 0.31 & \\
\hline \multirow[t]{2}{*}{$\begin{array}{l}\text { Factor III: loss of } \\
\text { confidence }\end{array}$} & $\begin{array}{l}\text { 10. Been losing } \\
\text { confidence in self }\end{array}$ & 264 & 0.27 & 199.5 \\
\hline & $\begin{array}{l}\text { 11. Been thinking of self } \\
\text { as worthless }\end{array}$ & 135 & 0.14 & \\
\hline
\end{tabular}

\section{Related factors of the mental health}

Multivariable logistic regression was performed to furtherly analyze the related factors for mental health status. As illustrated in Table 3, results of the multivariate analysis showed that male ( $\mathrm{OR}=1.573,95 \% \mathrm{Cl}$ : $1.385-1.853)$, work in closed jail $(\mathrm{OR}=2.203,95 \% \mathrm{Cl}: 2.139-2.297)$, more night shifts $(\mathrm{OR}=2.163,95 \%$ Cl: $2.031-2.317 ; \mathrm{OR}=2.749,95 \% \mathrm{Cl}: 2.194-2.901)$, more smoking $(\mathrm{OR}=1.100,95 \% \mathrm{Cl}: 1.037-2.168)$, poor physical condition ( $\mathrm{OR}=1.947,95 \% \mathrm{Cl}$ : 1.478-2.250), chronic or serious illness history $(\mathrm{OR}=1.870$, $95 \% \mathrm{Cl}: 1.314-2.660 ; \mathrm{OR}=2.214,95 \% \mathrm{Cl}: 1.460-2.812)$ were risk factor for mental health among frontline prison police, while regular diet $(\mathrm{OR}=0.779,95 \% \mathrm{Cl}: 0.539-0.928)$, more physical exercise (OR= $0.702,95 \% \mathrm{Cl}: 0.548-0.899 ; \mathrm{OR}=0.641,95 \% \mathrm{Cl}: 0.316-0.887$ ), more communication with family members (OR $=0.437,95 \% \mathrm{Cl}: 0.295-0.616)$ were protective factor. 
Table 3

Related factors for mental health through multivariate logistic regression analysis.

\begin{tabular}{|c|c|c|c|c|}
\hline Variables & Factors & $\beta$ & $\begin{array}{l}\mathrm{OR}(95 \% \\
\mathrm{Cl})\end{array}$ & $p$-value \\
\hline \multirow[t]{2}{*}{ Gender } & Female & Reference & 1.000 & - \\
\hline & Male & 0.557 & $\begin{array}{l}1.573 \\
(1.385- \\
1.853)\end{array}$ & $\star \star 0.006$ \\
\hline \multirow[t]{3}{*}{ Working years } & $<5$ & Reference & 1.00 & - \\
\hline & $5-10$ & - & - & $>0.05$ \\
\hline & $>10$ & - & - & $>0.05$ \\
\hline \multirow[t]{3}{*}{ Recent working mode } & $\begin{array}{l}\text { Home quarantine and } \\
\text { preparation }\end{array}$ & Reference & 1.000 & - \\
\hline & $\begin{array}{l}\text { Centralized isolation } \\
\text { and preparation }\end{array}$ & - & - & $>0.05$ \\
\hline & Work in closed jail & 1.592 & $\begin{array}{l}2.203 \\
(2.139- \\
2.297)\end{array}$ & $\begin{array}{l}* \star< \\
0.001\end{array}$ \\
\hline \multirow{3}{*}{$\begin{array}{l}\text { Number of night shifts } \\
\text { (times/month) }\end{array}$} & $<7$ & Reference & 1.000 & - \\
\hline & $7-15$ & 1.193 & $\begin{array}{l}2.163 \\
(2.031- \\
2.317)\end{array}$ & $\star 0.026$ \\
\hline & $>15$ & 1.225 & $\begin{array}{l}2.749 \\
(2.194- \\
2.901)\end{array}$ & $\begin{array}{l}* *< \\
0.001\end{array}$ \\
\hline \multirow[t]{2}{*}{ Diet } & irregular & Reference & 1.000 & - \\
\hline & regular & -0.405 & $\begin{array}{l}0.779 \\
(0.539- \\
0.928)\end{array}$ & $\star 0.031$ \\
\hline \multirow[t]{3}{*}{ Physical exercise (days/week) } & No exercise & Reference & 1.000 & - \\
\hline & $\leq 2$ & -0.354 & $\begin{array}{l}0.702 \\
(0.548- \\
0.899)\end{array}$ & $\star \star 0.005$ \\
\hline & $\geq 3$ & -0.626 & $\begin{array}{l}0.641 \\
(0.316- \\
0.887)\end{array}$ & $\begin{array}{l}* *< \\
0.001\end{array}$ \\
\hline
\end{tabular}

$\beta$. Standard partial regression coefficient, OR: Odd ratio, $95 \% \mathrm{Cl}$ : $95 \%$ confidence interval, ${ }^{\star \star}$ significant at $p<0.01$, *significant at $p<0.05$. 


\begin{tabular}{|c|c|c|c|c|}
\hline Variables & Factors & $\boldsymbol{\beta}$ & $\begin{array}{l}\text { OR }(95 \% \\
\mathrm{Cl})\end{array}$ & $p$-value \\
\hline \multirow[t]{3}{*}{ Smoking } & Never & Reference & 1.000 & - \\
\hline & Sometimes & - & - & $>0.05$ \\
\hline & Everyday & 0.550 & $\begin{array}{l}1.100 \\
(1.037- \\
2.168)\end{array}$ & *0.027 \\
\hline \multirow[t]{2}{*}{ Physical condition } & good & Reference & 1.000 & - \\
\hline & poor & 0.801 & $\begin{array}{l}1.947 \\
(1.478- \\
2.250)\end{array}$ & $\begin{array}{l}* *< \\
0.001\end{array}$ \\
\hline \multirow[t]{3}{*}{ Disease history in the past year } & No or minor illness & Reference & 1.000 & - \\
\hline & chronic & 0.626 & $\begin{array}{l}1.870 \\
(1.314- \\
2.660)\end{array}$ & $\star \star 0.001$ \\
\hline & serious illness & 1.217 & $\begin{array}{l}2.214 \\
(1.460- \\
2.812)\end{array}$ & $\begin{array}{l}* *< \\
0.001\end{array}$ \\
\hline \multirow{2}{*}{$\begin{array}{l}\text { Communication with family } \\
\text { members (times/week) }\end{array}$} & $0-2$ & Reference & 1.000 & - \\
\hline & $\geq 3$ & -0.354 & $\begin{array}{l}0.437 \\
(0.295- \\
0.616)\end{array}$ & $\begin{array}{l}* *< \\
0.001\end{array}$ \\
\hline
\end{tabular}

\section{Discussion}

The frontline healthcare workers and police staffs were always the major force that fighted the COVID-19 epidemic. Increasing attention has been paid on the mental health issues of frontline warriors during the global public health emergency $[5,13]$. However, the security personnel especially prison police who also actively engaged in preventing and controlling this epidemic were largely neglected. To the best of our knowledge, this study was firstly conducted to evaluate mental health status of frontline prison police in China during the COVID-19 epidemic, and to identify factors that influence psychological distress.

A total of 981 frontline prison police participated in the cross-sectional Web-based survey, which was considered large enough to draw definite inferences. The current study found that a $33.43 \%$ prevalence of psychological health issues among the police who prevented the COVID-19 epidemic from spreading to prison. The findings obtained in the study were higher than those obtained among healthcare professionals and the general population $(21.3 \%-33.0 \%)[15,24,25]$. There was no any data and 
previous research evaluating the mental health of prison police, resulting in a impossible comparison with the prevalence findings during other periods. However, respondents with prison police during the prevention and control of the COVID-19 epidemic reported significantly higher levels of psychological problems than those with Chinese adults surveyed in $2019(3.6 \%-5.0 \%)$ [26]. These results reflected that frontline prison police reached a level of mental health problems, which could not be ignored and required further evaluation.

The 12-item General Health Questionnaire (GHQ-12) was an effective instrument for screening mental disorders, and has been widely used in Chinese community samples [17, 20]. The three-factor model of GHQ-12 proposed by Worsley and Gribbin (1977) [22], including anxiety and depression, social dysfunction, and loss of confidence, was verified to give the best fit [23]. Psychological factors analysis showed that frontline prison police mainly experiencd mental health issues associated with anxiety and depression, and issues related to these symptoms were unhappy and depressed, lost sleep over worry and constantly under strain. These findings were consistent with several researches, which suggested that anxiety, depression, sleep issues [27], and stress [28] were the most common psychological problems caused by COVID-19 epidemic. Excessive anxiety might lead to several harmful consequences including lower quality of life [29], suicidal ideation and attempts [30], physical chronic conditions [31] and relationship complications [32]. Many emotional and physical health problems, as well as decreasion of the ability to function at work and home, could be elicited by depression [33, 34]. It implied that psychological intervention should be carried out for frontline prison police early to help alleviate their psychological symptoms.

Despite it was necessary to evaluate the prevalence of psychological symptoms in frontline prison police, the further analysis for identifying risk and protective factors contributing to mental health issues was more importantly. Data from the present study showed that male frontline prison police was more likely to suffer from mental health problems compared with female prison police. This contrasted with findings in the general population [35], healthcare workers [36], and police personnel [13] during the COVID-19 epidemic. Reason for the finding was attributed to men might take more responsibility leading to psychological distress symptoms [37]. The participants with work in closed jail recently were about 2.203 times $(O R=2.203)$ more likely to have mental health problems than that with home quarantine and preparation, which might be because a person in a closed environment could develop psychological problems [38]. Our results suggested that more night shifts have increased the risk of mental health problems in frontline prison police. Consistent with earlier study, more night shifts could cause sleep disturbances, and burnout and mental disorder among employees [39]. The frontline prison police with tendency of mental health problems smoked significantly more and were therefore at greater risk [40]. Moreover, participants who were poor physical condition and chronic or serious illness history had a higher risk of psychological issues. It might indicate that mental health defense of this prison police subgroup with weak physical function has been negatively affected.

Previous studies have confirmed that healthy diets and exercise regularly could promote mental health for the adult population $[41,42]$. Our data proved that regular diet and more physical exercise were 
protective factors for mental health among frontline prison police, and the incidence of psychological problems were 0.779 and 0.702 times that of the opposite. The present study also revealed that comunication with family members was another protective factor associated with mental health of the prison police during the prevention and control of the COVID-19 epidemic. This might be due to the fact that providing emotional support from family and friends and maintaining contact were positive treatment to protect mental health [43].

Since the early phase of COVID-19 epidemic control, the prison authorities in China have been constantly implemented psychological interventions, including published the mental health handbook for prison police, established psychological assistance hotlines, and assigned mental health professionals to provide timely psychiatric help. These measures have efficiently alleviated the psychological problems of frontline prison police, thereby reducing the rate of work errors. As the normalization of epidemic prevention and control will continue for a long time, this study may provide practical guidance for the development of a psychological support strategy. According to the current findings, the targeted interventions should be performed to relieve mental health problems among frontline prison police. First, prison authorities should formulate and implement a viable strategy to adjust duty hours to the optimization, and provide solid logistical support to guarantee prison police' quality of life with sleep, diet and exercise, so that their physical and mental health problems can be reduced. Second, the mental health of frontline prison police should be dynamically monitored to provide appropriate information on treatment and psychological intervention for affected people, and supply continuity of regular mental health care services among the whole population. Third, the prison police should maintain a good living habits to promote mental health, and learn to use emotion regulation strategies for reducing psychological stress. Fourth, the authorities need to emphasize the role of family support during this epidemic, increase opportunities for frontline prison police to communicate with family members, so as to avoid their psychological disorders caused by closed information between each other. Fifth, our government and relevant authorities should strengthen the psychological evaluation, online psychological counseling, psychological crisis prevention and intervention of frontline prison police during epidemic control and in the future.

There have been several limitations in present study, which were as follows: (1) our respondents were all from China, which might not fully represent the mental health status of frontline prison police around the world. (2) Given the particularity and complexity of COVID-19 epidemic prevention and control, we have conducted an online survey using snowball sampling approach, which the participants were limited to using smartphones or computers with network link service. Moreover, the prison police on duties might be overworked, leading to the consequence that many shared links were not responded. (3) The mental health problems of frontline prison police might have been biased due to recall and selection bias in selfreports on the individual's physical and mental health. (4) This study have employed cross-sectional design, so only association analyses allowed and causal inferences could not be made, and it only revealed the psychological status of frontline prison police during a certain period of epidemic control. Future research should adopt longitudinal design to evaluate the long-term psychological implications of the epidemic through exploring multiple time points. 


\section{Conclusion}

To sum up, the present study indicated that mental health problems were serious among frontline prison police during the prevention and control of the COVID-19 epidemic. This study also suggested that male, work in closed jail, more night shifts, more smoking, poor physical condition, chronic or serious illness history, regular diet, more physical exercise, more communication with family members were the influence factors of mental health. These findings highlighted that the need for prison authorities to continuously monitor the psychological impact of public health emergency on frontline prison police, and timely develop and implement targeted interventions to tackle mental health problems.

\section{Abbreviations}

COVID-19

Coronavirus Disease 2019; GHQ-12:the 12-item General Health Questionnaire; OR:odds ratios; 95\% $\mathrm{Cl}: 95 \%$ confidence interval

\section{Declarations}

\section{Acknowledgements}

We would like to thank all of participates for their great support which made this study possible. We would also like to express our gratitude to the frontline medical and police staff who fought the pandemic, and sympathy for the victims during the COVID-19 pandemic.

\section{Authors' contributions}

YT and JH contributed to the design, analysis, drafted the manuscript, critically revised the manuscript, and gave final approval. TF contributed to design, modify and revise the manuscript. YY contributed to analysis, drafted and revise the manuscript. ZW contributed to the draft and gave final approval. All authors have read and approved the final manuscript.

\section{Funding}

This article did not receive any specific grant from funding agencies in the public, commercial, or any other sectors.

\section{Availability of data and materials}

The study data used and analyzed in this study are available from the corresponding author on reasonable request.

\section{Ethics approval and consent to participate}


This study protocol was approved by the Ethical Review Board of West China Medical School at Sichuan University. An online written informed consent was provided from all participates, and the survey questions were answered anonymously.

\section{Consent for publication}

Not applicable

\section{Competing interests}

All authors declare no conflict of interest.

\section{Author details}

${ }^{1}$ Research Center of Criminal Execution and Prosecution, Renmin University of China, BeiJing 100872, PR China. ${ }^{2}$ Research center of Psychology and Social Develoment, Faculty of Psychology, Southwest University, Chongqing 400715, PR China. ${ }^{3}$ Department of General Surgery, Chengdu Third People's Hospital, Chengdu 610031, PR China. ${ }^{4}$ Department of Judicial Information Management, Sichuan Judicial and Police Officers Peofessional College, DeYang 618000, PR China. ${ }^{5}$ West China Medical School College, Sichuan University, Chengdu 610041, PR China.

\section{References}

1. WHO. Statement on the second meeting of the International Health Regulations (2005) Emergency Committee regarding the outbreak of novel coronavirus(2019-nCoV). 2020. https://www.who.int/news-room/detail/30-01-2020-statement-on-the-second-meeting-of-theinternational-health-regulations-(2005)-emergency-committee-regarding-the-outbreak-of-novelcoronavirus-(2019-ncov). Accessed 30 Jan 2020.

2. WHO. Coronavirus disease (COVID-19) outbreak. 2020. https://www.who.int/ emergencies/diseases/novel-coronavirus-2019. Accessed 26 Jan 2021.

3. Nidal D, Ayat A, Hana AIN, Mahra A, Shaikha A, Sofia O. Investigating the Impact of COVID-19 Lockdown on the Psychological Health of University Students and Their Attitudes Toward Mobile Mental Health Solutions: Two-Part Questionnaire Study. JMIR Form Res. 2020; 4(10):e19876.

4. Doyle TJ. COVID-19, Prison Visits, and the Value of a Cup of Coffee. Ann Intern Med. 2020;173(8): 666-7.

5. Muller AE, Hafstad EV, Himmels JPW, Smedslund G, Flottorp S, Stensland S $\emptyset$, et al. The mental health impact of the covid-19 pandemic on healthcare workers, and interventions to help them: $A$ rapid systematic review. Psychiatry Res. 2020; 293: 113411.

6. Amin F, Sharif S, Saeed R, Durrani N, Jilani D. COVID-19 pandemic- knowledge, perception, anxiety and depression among frontline doctors of Pakistan. Br J Psychiatry. 2020; 20:459. 
7. Edge C, Hayward A, Whitfield A, Hard J. COVID-19: digital equivalence of health care in English prisons. The Lancet Digital Health. 2020; 2(9): e450-9.

8. Fovet T, Lancelevee C, Eck M, Scouflaire T, Becache E, Dandelot D, Giravalli P, Guillard A, Horrach P, Lacambre M, Lefebvre T, et al. Mental health care in French correctional facilities during the Covid-19 pandemic. ENCEPHALE. 46(3): S60-5.

9. Saloner B, Parish K, Ward JA, Dilaura G, Dolovich S. COVID-19 Cases and Deaths in Federal and State Prisons. J Am Med Assoc. 2020; 324(6): 602-3.

10. Dmello JR, Ranjan S. Lock Unlock: The Impact of COVID-19 on Health Security in Pakistani and Indian Prisons. Victims \& Offenders. 2020; 15(2): 970-97.

11. Ribeiro L, Diniz AMA. The Brazilian Penitentiary System under the Threat of COVID-19. Victims \& Offenders. 2020; 15(7-8): 1019-43.

12. Grover S, Sahoo S, Dua D, Mehra A, Nehra R. Psychological Impact of COVID-19 Duties During Lockdown on Police Personnel and Their Perception About the Behavior of the People: an Exploratory Study from India. Int J Ment Health Ad. 2020; 12 - 5. https://doi.org/10.1007/ s11469020-00408-8.

13. Li L, Wan C, Ding R, Liu Y, Chen J, Wu Z, et al. Mental distress among Liberian medical staff working at the China Ebola treatment unit: a cross sectional study. Health Qual Life Outcomes. 2015;13(1):156.

14. Pappa S, Ntella V, Giannakas T, Giannakoulis VG, Papoutsi E, Katsaounou P. Prevalence of depression, anxiety, and insomnia among healthcare workers during the COVID-19 pandemic: A systematic review and meta-analysis. Brain Behav. Immun. 2020; 88: 901-7.

15. Zhang J, Wu W, Zhao X, Zhang W. Recommended psychological crisis intervention response to the 2019 novel coronavirus pneumonia outbreak in China: A model of West China Hospital. Precision Clinical Medicine. 2020; 3: 3-8.

16. Cheng TA, Wu JT, Chong MY, Williams P. Internal consistency and factor structure of the Chinese Health Questionnaire. Acta Psychiatr Scand. 1990; 82: 304-8.

17. Jin Y, Zhang YS, Zhang Q, Rao W, Xiang YT. Prevalence and Socio-Demographic Correlates of Poor Mental Health Among Older Adults in Agricultural Areas of China. Front Psychiatr. 2020; DOI: 10.3389/fpsyt.2020.549148.

18. Liang L, Ren H, Cao R, Hu Y, Qin Z, Li C, Mei S. The effect of COVID-19 on youth mental health. Psychiat Quart. 2020; 91: 841-52.

19. Yang TZ, Huang L, Wu ZY. The application of Chinese health questionnaire for mental disorder screening in community settings in mainland China. Chin J Epidemiol. 2003; 24: 769-73.

20. Wang W, Ding L, Liao Z. The best thresholds and the screening features among the three scoring methods of the 12-item General Health Questionnaire. Chin J Psychiatry. 2012; 45: 349-53.

21. Worsley A, Gribbin CC. A factor analytic study on the twelve item general health questionnaire. Aust $N$ Z J Psychiatry. 1977; 11(4): 260-72. 
22. Mäkikangas A, Feldt T, Kinnunen U, Tolvanen A, Kinnunen M, Pulkkinen L. The factor structure and factorial invariance of the 12-item General Health Questionnaire (GHQ-12) across time: evidence from two community-based samples. Psychol Assess. 2006;18(4): 444-51.

23. Cao W, Fang Z, Hou G, Han M, Xu X, Dong J, et al. The psychological impact of the COVID-19 epidemic on college students in China. Psychiatry Research. 2020; 287: 112934.

24. Luo M, Guo L, Yu M, Wang $\mathrm{H}$. The psychological and mental impact of coronavirus disease 2019 (COVID-19) on medical staff and general public-A systematic review and meta-analysis. Psychiatry Res. 2020; 291: 113190.

25. Huang $Y$, Wang $Y$, Wang $H$, Liu Z, Yu X, Yan J, et al. Prevalence of mental disorders in China: a crosssectional epidemiological study. Lancet Psychiatry. 2019; 6(3): 211-24.

26. Huang Y, Zhao N. Generalized anxiety disorder, depressive symptoms and sleep quality during COVID-19 outbreak in China: a web-based cross-sectional survey. Psychiatry Res. 2020; 288: 112954.

27. Kar N, Kar B, Kar S. Stress and coping during COVID-19 pandemic: Result of an online survey. Psychiatry Res, 2021; 295: 113598.

28. Brenes GA. Anxiety, depression, and quality of life in primary care patients. Prim Care Companion J Clin Psychiatry. 2007; 9(6): 437-43.

29. Nepon J, Belik SL, Bolton J, et al. The relationship between anxiety disorders and suicide attempts: findings from the National Epidemiologic Survey on Alcohol and Related Conditions. Depress Anxiety. 2010; 27(9): 791-8.

30. Sareen J, Cox BJ, Clara I, et al. The relationship between anxiety disorders and physical disorders in the US National Comorbidity Survey. Depress Anxiety. 2005; 21(4): 193-202.

31. Kasalova P, Prasko J, Holubová M, et al. Anxiety disorders and marital satisfaction. Neuro Endocrinol. Lett. 2017; 38(8), 555-64.

32. Hawton $\mathrm{K}$, Comabella CC, Haw C, et al. Risk factors for suicide in individuals with depression: a systematic review. J Affect Disord. 2013; 147(1-3): 17-28.

33. American Psychiatric Association. What Is Depression? 2020. https://www. psychiatry. org /patientsfamilies/depression/what-is-depression.

34. Jiang W, Liu X, Zhang J, Feng Z. Mental health status of Chinese residents during the COVID-19 epidemic. Br J Psychiatry. 2020; 20:580.

35. Liu Y, Liu X, Gao B, Li C, Liang XS. Mental distress among frontline healthcare workers outside the central epidemic area during the novel coronavirus disease (COVID-19) outbreak in China: A crosssectional study. COVID-19 research community. https: //doi.org/10.21203/rs.3.rs-32833/v1.

36. Guo J, Fu MQ, Xing J, Qu ZY, Wang XU. Coping style and posttraumatic growth among adult survivors 8 years after the 2008 Wenchuan earthquake in China. Pers Indiv Differ. 2017; 111: 31-6.

37. Klochko YV. A person in a closed environment as a psychological problem. Psychology in Russia: State of the Art. 2013; 6(4):143. 
38. Cheng W J, Cheng, Y W. Night shift and rotating shift in association with sleep problems, burnout and minor mental disorder in male and female employees. BJM Case reprots. 2016; 74(7): 483-8.

39. Campion J, Checinski K, Nurse J, McNeill A. Smoking by people with mental illness and benefits of smoke-free mental health services. Advances in Psychiatric Treatment. 2008; 14(3): 217-28.

40. Roohafza H, Sarrafzadegan N, Sadeghi M, Rafieian-Kopaei M, Sajjadi F, Khosravi-Boroujeni H. The association between stress levels and food consumption among Iranian population. Arch Iran Med. 2013; 16(3): 145-8.

41. Kenari MA. Effect of Exercise on Mental Health in the Physical Dimension, Anxiety and Mental Disorder, Social Dysfunction and Depression. AASoci. 2014; 4(3): 63-8.

42. Morgan AJ, Reavley NJ, Jorm AF, Beatson R. Discrimination and support from friends and family members experienced by people with mental health problems: findings from an Australian national survey. Soc Psych Psych Epid. 2017; 52(11): 1395-403.

\section{Figures}

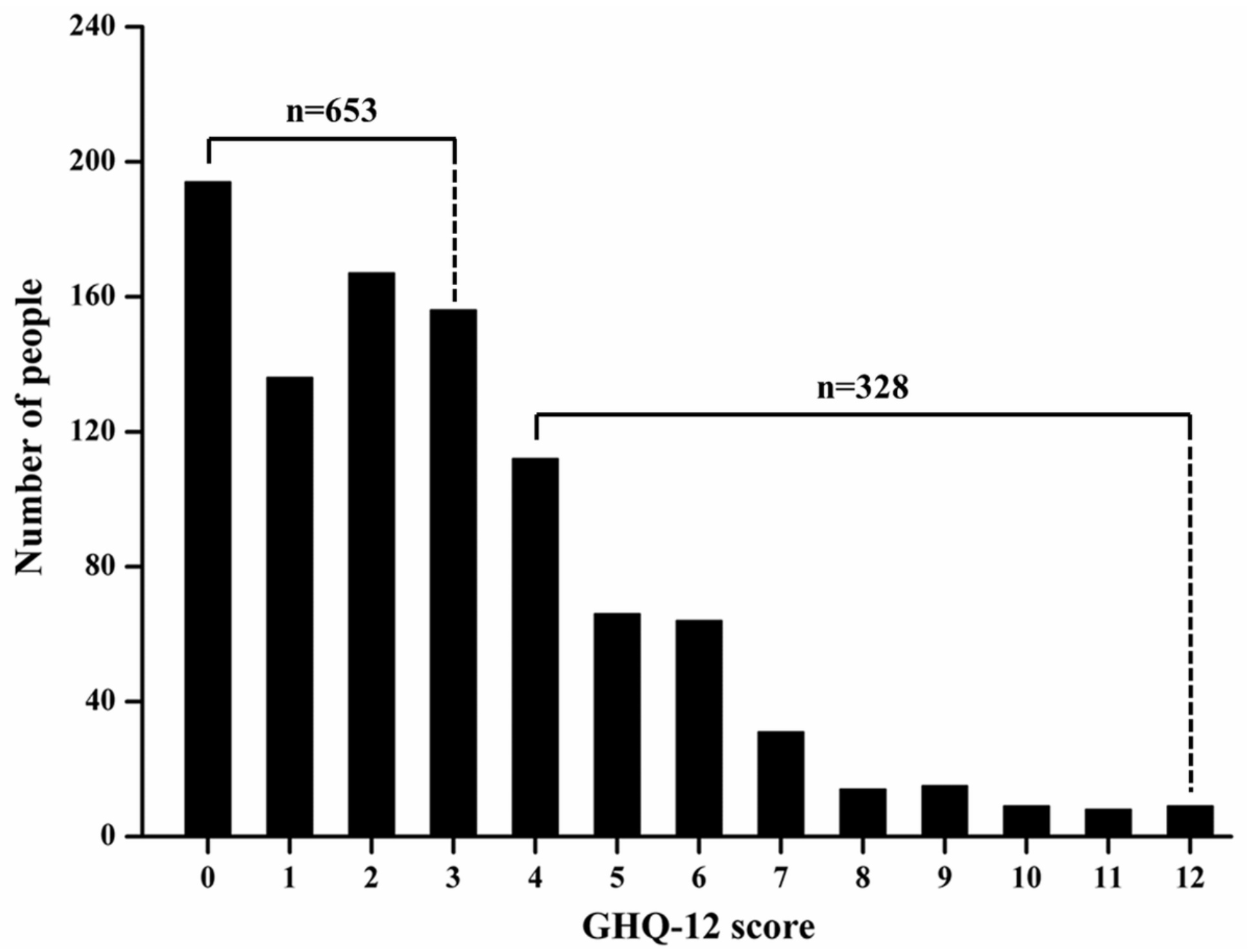




\section{Figure 1}

The self-reported mental health status of frontline prison police by GHQ-12 score distribution.

\section{Supplementary Files}

This is a list of supplementary files associated with this preprint. Click to download.

- Supplementarymaterial.docx 